\section{Diagnosis and management of lysosomal storage disorders. Three key words: early, multidisciplinary, and network}

\author{
Generoso Andria, ${ }^{1}$ Giuseppe Limongelli ${ }^{2}$ \\ 'Department of Translational Medical \\ Sciences, Federico II University; ${ }^{2}$ Second \\ University of Naples, Monaldi Hospital, \\ Naples, Italy
}

The European Commission on Public Health defines as rare diseases life-threatening or chronically debilitating diseases which are of such low prevalence that special combined efforts are needed to address them. ${ }^{1}$ The term low prevalence is taken as prevalence of less than 5 per 10,000 in the European Union. The definition is slightly different in the US (any disease or condition that affects less than 200,000 persons in the United States, or about 1 in 1500 people, according to the Rare Disease Act of 2002) and in Japan (one that affects fewer than 50,000 patients in Japan, or about 1 in 2500 people)..$^{2}$ It is interesting to note that epidemiological data on rare disorders are rapidly changing with the new screening technology presently available. Indeed, in this Special issue of the journal, Cecchi et al. points out that new epidemiological data based on newborn screening showed that the prevalence of Anderson-Fabry disease (AFD) is higher than previously reported. ${ }^{3-5}$

The umbrella rare disease includes genetic syndromes, inherited metabolic diseases (inborn error or metabolism) and neuromuscular disorders with various organ involvement and phenotypic expression. In most cases, a multidisciplinary approach is required in the diagnosis and management of the diseases. The diagnosis is generally difficult and is often delayed, since the clinical characteristics of the disease are not well recognized by most of the clinicians. However, in the last few years, attention is growing due to the interest for innovative therapeutical approaches. Lysosomal storage disorders (LSD), i.e. a group of disorders characterized by various enzymatic deficits regulating the metabolism of glycogen, mucopolysaccharides and sphyngolipids, is a classical example. For a long time, these disorders have been frustrating for clinicians and a death sentence for patients, while in the last 20 years the discovery of new therapies (i.e. enzyme replacement therapy, ERT) has given the opportunity to clinicians to change the natural history of LSD. The cardiologist is one of the specialists involved in LSD management. Indeed, myocardial involvement (cardiomyopathy) is a typical feature of Pompe disease and AFD, while valvular degeneration is typically present in patients with mucopolysaccharidoses $^{6}$ and, sometimes, in patients with Gaucher disease. ${ }^{7}$ Early diagnosis is one of the key points for an efficacious ERT, and this is a fundamental message for any specialist involved in the management of these disorders. The knowledge and early clinical recognition of the classical clinical features of the disease (red flags) are essential for the diagnosis. ERT in patients with early symptoms and initial organ disease, such as microalbuminuria and neuropathic pain, seem to be effective in AFD. $^{3}$ A presentation with severe muscle involvement (floppy baby) and progressive cardiomyopathy in the newborn should immediately arise the suspect of a metabolic disorder, potentially treatable, as Pompe disease. The presence of conduction abnormalities at electrocardiogram and macroglossia represents cardiac and non-cardiac red flags, respectively. ${ }^{8}$ It is important to remember that some of the red flags are age-related, as proteinuria and cardiomyopathy in AFD (rarely present in young patients). ${ }^{3}$

In this issue of Cardiogenetics, we aimed to describe the genetic basis, the multidisciplinary diagnosis and the management of the principal LSD. To this purpose, we invited a group of specialists for each disease to describe their day-by-day clinical experience with LSD, and their experience to co-operate as a network. In the near future new therapeutic approaches are about to happen (gene therapy chaperones, etc.), ${ }^{9,10}$ but the main secret for the diagnosis and management remains in the three key words: early, multidisciplinary, network.

\section{References}

1. European Commission, Health and Consumer Protection Directorate. Useful information on rare diseases from an EU perspective. Available from: http//ec.europa. eu/health/ph_information/documents/ev200 40705_rd05_en.pdf

2. Wikipedia. Rare disease. Available from:
Correspondence: Generoso Andria, Department of Translational Medical Sciences, Federico II University, Via S. Pansini 5, 80131 Naples, Italy.

E-mail: andria@unina.it

Key words: lysosomal storage disorders, diagnosis and management.

Received for publication: 8 February 2013. Accepted for publication: 11 February 2013.

This work is licensed under a Creative Commons Attribution NonCommercial 3.0 License (CC BY-NC 3.0).

(C) Copyright G. Andria and G. Limongelli, 2013 Licensee PAGEPress, Italy

Cardiogenetics 2013; 3(s1):e1

doi:10.4081/cardiogenetics.2013.s1.e1

http://en.wikipedia.org/wiki/Rare_disease

3. Cecchi F, Tomberli B, Morrone A. Anderson-Fabry, the histrionic disease: from genetics to clinical management. Cardiogenetics 2013;3(s1):e3.

4. Lin H-Y, Chong K-W, Hsu J-H, et al. High incidence of the cardiac variant of Fabry disease revealed by newborn screening in the Taiwan Chinese population. Circ Cardiovasc Genet 2009;2:450-6.

5. Spada M, Pagliardini S, Yasuda M, et al. High incidence of later-onset fabry disease revealed by newborn screening. Am J Hum Genet 2006;79:31-40.

6. Parini R, Bertola F, Russo P. Molecular basis, diagnosis and clinical management of mucopolysaccharidoses. Cardiogenetics 2013;3(s1):e2.

7. Di Rocco M, Loggini A, Russo P. Molecular basis and clinical management of Gaucher disease. Cardiogenetics 2013;3(s1):e4.

8. Parenti G, Di Iorio G, Sampaolo S, et al. Molecular basis and clinical management of Pompe disease. Cardiogenetics 2013;3 (s1):e5.

9. Sardiello M, Palmieri M, di Ronza A, et al. A gene network regulating lysosomal biogenesis and function. Science 2009;325: 473-7.

10. Benjamin ER, Khanna R, Schilling A, et al. Co-administration with the pharmacological chaperone AT1001 increases recombinant human $\alpha$-galactosidase A tissue uptake and improves substrate reduction in Fabry mice. Mol Ther 2012;20:717-26. 